\title{
The CDEN e-Design Portal
}

\author{
Robert W. Brennan \\ Department of Mechanical and Manufacturing Engineering \\ University of Calgary, 2500 University Dr. N.W., Calgary, AB, T2N $1 N 4$ \\ Phone: +1-403-220-5798 \\ Email: rbrennan@ucalgary.ca
}

\begin{abstract}
In this paper we provide an overview of the current work that is being conducted to develop a single point of entry or "e-Design Portal" for engineering design courseware for the Canadian Design Engineering Network (CDEN). The general architecture of the $e$ Design Portal is described and examples of its use with existing CDEN modules are provided.
\end{abstract}

\section{Introduction}

Central to the Canadian Design Engineering Network (CDEN) is the notion that the internet will be used to deliver design-directed learning [1] modules to improve engineering design competency in future graduates [2]. Although it is expected that CDEN module developers are experts in the content of their respective modules, their degree of expertise in the area of e-learning and, in particular, web-based teaching of open-ended design will vary considerably. Despite this, the success of the CDEN network ultimately rests on the quality, usability, and accessibility of these modules.

In this paper, we focus on the accessibility of CDEN modules. More specifically, we describe the initial design of the web server that will be used to manage the submission, searching, and retrieval of CDEN modules.

The paper begins with an overview of the basic requirements for this web server. These requirements came out of a workshop on CDEN modules that was held in October 2004 [3]. Next, we look at a general architecture for the CDEN server that can be used to meet these requirements. This approach is currently being used to develop a single point of access for CDEN design modules. We follow this with a section on the current implementation of the web server with examples of existing modules from the CDEN community. The paper concludes with a summary of our current work on the development of the CDEN server.

\section{The Web Server Requirements}

The requirements for the CDEN web server were recently tackled at a workshop of CDEN module developers [3]. At this workshop, module developers identified organization and module search-ability as key characteristics of the portal. As well, it was felt that the portal should include a means to allow users to provide feedback on the strengths and weaknesses of modules.

More specifically, the question "what overall structure should the CDEN website ultimately have, and what is the process to co-opt the engineering disciplines and define this?" was discussed. To address the question of "overall structure", the workshop participants focused on the desired characteristics of the website and the website content.

In terms of desired characteristics, the following points were made about the future web server:

- it must be well-organized with respect to architecture via key words (discipline, competency, tier, design topic, design tool...),

- it must have a user-friendly search vehicle,

- it should be bilingual (as much as possible),

- it should monitor hits by module,

- it should permit feedback re strengths and it weaknesses of modules, and

- it should cater to early year design as well as capstone design.

The participants felt that the web server should contain the following basic content:

- a discussion of the undergraduate curriculum and the design competency associated with 
each discipline, including insight re how this competency can be taught,

- a discussion of the objectives, standards, considerations related to the modules,

- a directory of modules structured in the CDEN format

- other web-based design materials that is of any format,

- examples of design tools specific to and common to different engineering disciplines

- well-organized design information sources,

- protected and unprotected website areas, and

- material that is generic design, material specific to engineering disciplines, material that involves several disciplines.

In order to define what the website structure might include it was felt that CDEN's truly multidisciplinary nature needs to be captured and the website should be inclusive of every department of every engineering faculty in Canada. The process to define this must necessarily be bottom-up, involving every discipline.

Associated with the website there should be activities/expectations which promote understanding of the design competency. Once the website has value to offer, CDEN needs to have a proactive marketing plan including a description of the state-of-the-art and the vision. As well, an ongoing benchmarking activity is required.

\section{The e-Design Portal Architecture}

Courseware is not new to engineering education. In fact, the name "courseware" only implies that engineering course material is now in an electronic form (rather than paper notes). Other than the ability to incorporate media elements (e.g., audio and visual techniques) the content basically remains the same. As well, a strong case can be made that it is the instructional methods and not the medium that causes learning; however, each medium offers unique opportunities to deliver instructional methods that other media cannot [4].

In the case of engineering design education, we feel that an e-learning approach can be used to help manage the complexity of typical design problems by helping students to through different levels or "tiers" [5] as well as different stages of the problem.

In this section we describe our current work on developing a web-based interface, or "e-Design Portal", that is intended to be used to show the interrelationships between engineering course material in the context of real engineering design problems. It should be noted that although the majority of the modules that will be added to the e-Design Portal have been tested in classroom settings by their authors, the notion of a single point of entry for a variety of modules is new and remains to be evaluated.

To accomplish this, "topics" and CDEN Tiers are being used to provide the website with a sense of structure and context. The portal's front page features a given topic or tier, as well as highlight various modules of interest, whether they are the newest resources to be added, or the most popular resources at that time. This general structure is shown in Figure 1.

From this front page, users will be able to view pages for each tier and topic. These pages will feature editorial content that further contextualizes the materials in a magazine-like format. There will also be a way for users to contribute to discussions on that topic or tier, providing a means for them to build their own context.

To allow users to subscribe to a topic's modules and/or discussions and be notified as new items are available, RSS (Really Simple Syndication) [6] is being used for lists of modules and discussions (i.e., the "XML" links shown in Figure 1). This will also allow users to reuse this content in other places (e.g., within Blackboard courses) [7] or on external websites.

The modules used throughout the tiers and topics will have various relationships to each other. For example, a topic might have several Tier 1 modules that can be inferred to be related by topic. These relationships will be used to help guide instructors and students to various modules that can be used to support their teaching and learning, without forcing them to perform additional searches to find these resources. A user would be able to select a module to view more information about it and be provided with a list of other modules that are used in the same tier and/or topic as the selected one.

Although metadata will be important to enable efficient indexing and retrieval of modules, the focus will be shifted from metadata to the modules themselves for users. For example, users will be provided with a guided interface for metadata entry which would ask the user questions about the module being added and combine that with an analysis of the module itself (both the file and its contents) when building the metadata description of the module.

As noted previously, modules are organized in three CDEN Tiers. Given the nature of this classification, modules from each tier will have different needs with respect to design and implementation. The author is currently working on a set of guidelines [8] for the development of CDEN modules; in particular, the guidelines will propose three distinct but related templates to be used, one for each content type. Tier 1 


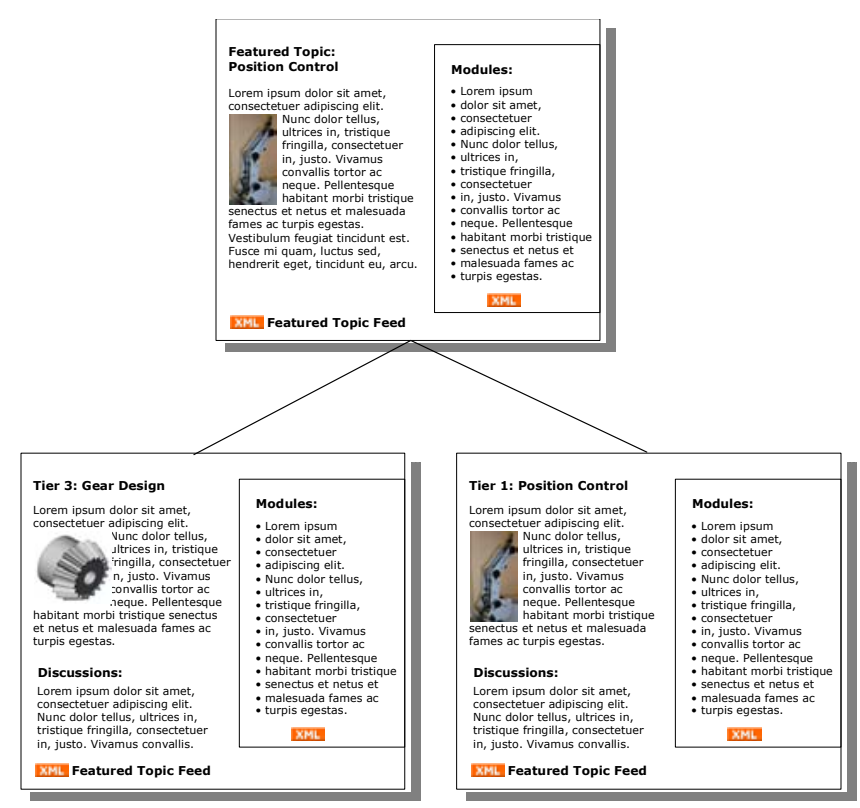

Figure 1. The General Structure of the e-Design Portal

modules will be mostly expository and highly textual in nature, with dense content and little need for user interaction. The module design for this might be more similar to a textbook chapter. Tier 2 are more descriptive of real-world applications of engineering science and technology as will need to be more flexible in the types of content that are available, as well as providing some opportunity for users to discuss and review the case study.

Finally, the Tier 3 modules will provide little content themselves, but must invite the users to publish and share their own content about the provided inquiry topic. Threaded discussion tools, weblogs, or collaborative wikis might be appropriate for inclusion within a Tier 3 module; this would allow the users to easily contribute throughout the process of research and inquiry.

\section{Using the e-Design Portal}

A prototype version of the CDEN e-Design Portal [9] is currently being developed by the author in conjunction with the University of Calgary's Learning Commons [10]. The front page interface is shown in Figure 2.

As noted previously, users have the option of viewing modules by "topics" or "tiers" as shown in the upper right part of the window in blue and yellow respectively. Additionally, users may search for modules using keywords that are indexed on the modules' textual information and metadata.

The front page is also used to feature any aspect of the e-Design Portal such as individual modules, topics, and/or discussions. In this case, three modules are featured: "Flexible Cut-off Saw Linear/Rotational Stage Design", "Design for the Environment", and "Safety Standards for Children".

The view of the "Inspect Module Topics" page is shown in Figure 3. At the time of writing, five "topics" were identified for the e-Design Portal: "Product Development Process", "Flexible Cut-off Saw System”, "Children's Sensory Simulation (CSS) Centre", "Linkages for Motion Generation", and "Engineering Case Studies".

As can be seen in Figure 3, users have the choice of viewing the modules that fall under each topic or joining a discussion on the topic (i.e., the buttons on the right of each topic). For example, the "Flexible Cut-off Saw System" topic contained 3 modules at the time of writing. When "view 3 modules" is selected, the page shown in Figure 4 appears.

For this example, the three modules that fall under the "Flexible Cut-off Saw System" topic are listed as shown in Figure 4 with a brief description of each module as well as options to either view the module or discuss it. As well, the topic description is provided at the top of the page. 


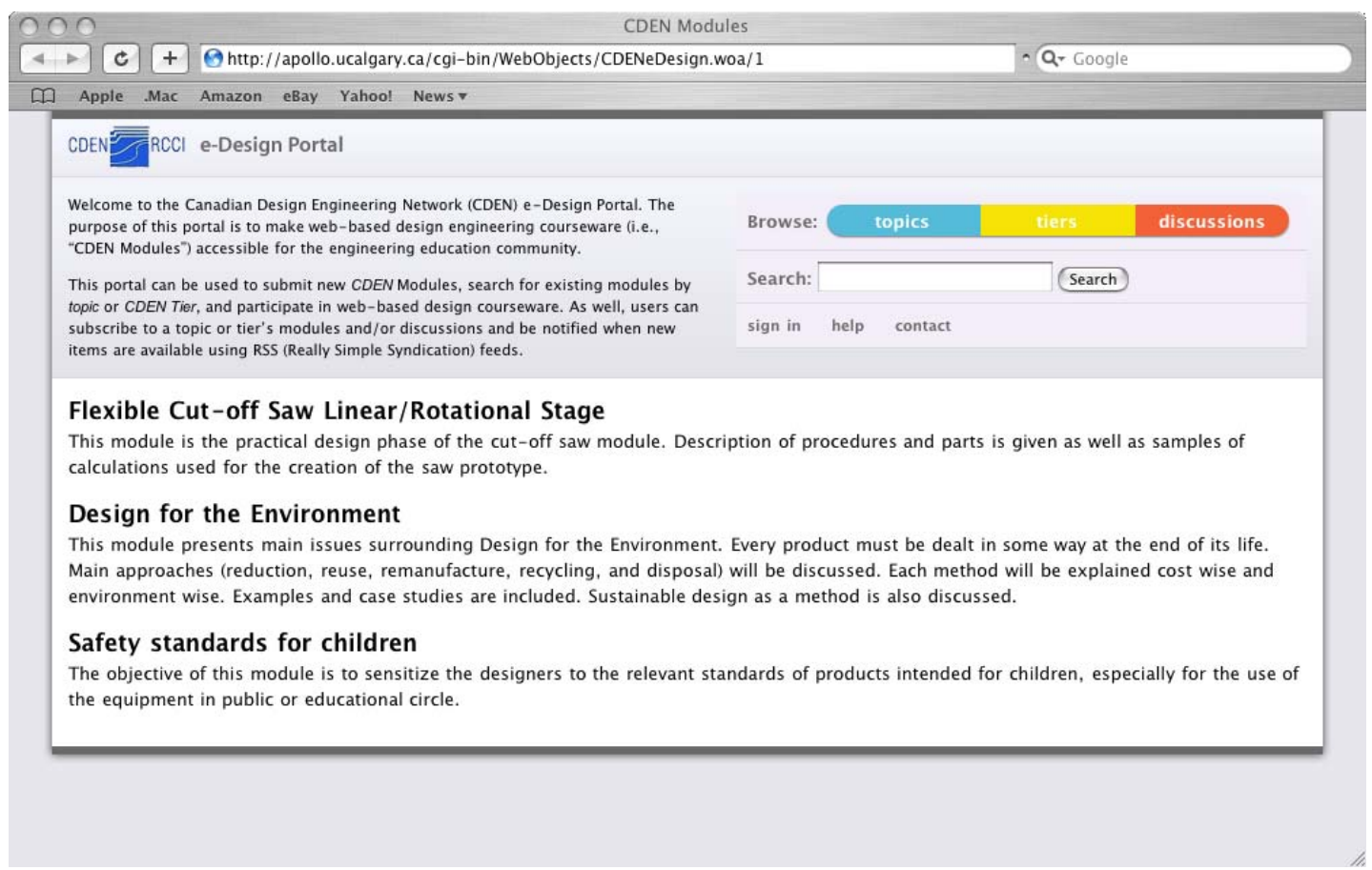

Figure 2. e-Design Portal front page

In addition to viewing modules by "topic", it is also possible to view modules by CDEN tier [5] as shown in Figure 5. It should be noted at this point that users are provided with a simple visual cue that helps identify how they are indexing modules in the eDesign Portal. For example, pages concerned with "topic" searches have a blue upper border as shown in Figures 3 and 4; pages concerned with "tier" searches have a yellow upper border as shown in Figure 5; and pages concerned with discussions have a red border.

As shown in Figure 5, modules are indexed by CDEN tier. In this case, the description of each tier is summarized from Yellowley et al. [5] and provided on the page. As well, the name of each tier has been modified slightly by the author to reflect the nature of the modules available at the present time.

Finally, users of the e-Design Portal have the opportunity to enter new CDEN design modules via the "upload" interface shown in Figure 6. This interface can be accessed by first signing into the eDesign Portal, then accessing "upload" from any page in the e-Design Portal.

In addition to the expected information such as the module's name, author, and description (abstract), certain basic information must be supplied as a standard before the module can be uploaded to the eDesign Portal. In particular, the module developer should state the module's relationship to the CDEN Tiers, any prerequisite competencies required for the module, and the module's relationship to existing topics.

This first point is important to the general organization of the e-Design Portal as illustrated in Figures 3-5, while the second point relates to module indexing and search. In particular, it is important that each module is provided with appropriate metadata to enable searching and indexing.

Since the teaching of engineering design competency is a key issue, it was felt that the metadata should be organized around the notion of design competencies [2] to allow users to search and retrieve modules on this basis. As can be seen in the lower half of Figure 6, users are required to select the competencies from seven categories before uploading their module: "General Knowledge", "Specific Knowledge", "Knowledge of Procedures", "Operational Skills", "Experiential Skills", "Cognitive Skills", and "Social/Personal Skills". This metadata is then used to index and ultimately enable search and retrieval of modules referenced in the e-Design Portal.

\section{Conclusions and Future Work}

In this paper we have provided an overview of the current work that is being conducted by the Canadian Design Engineering Network (CDEN) to develop a single point of entry or "e-Design Portal" for 


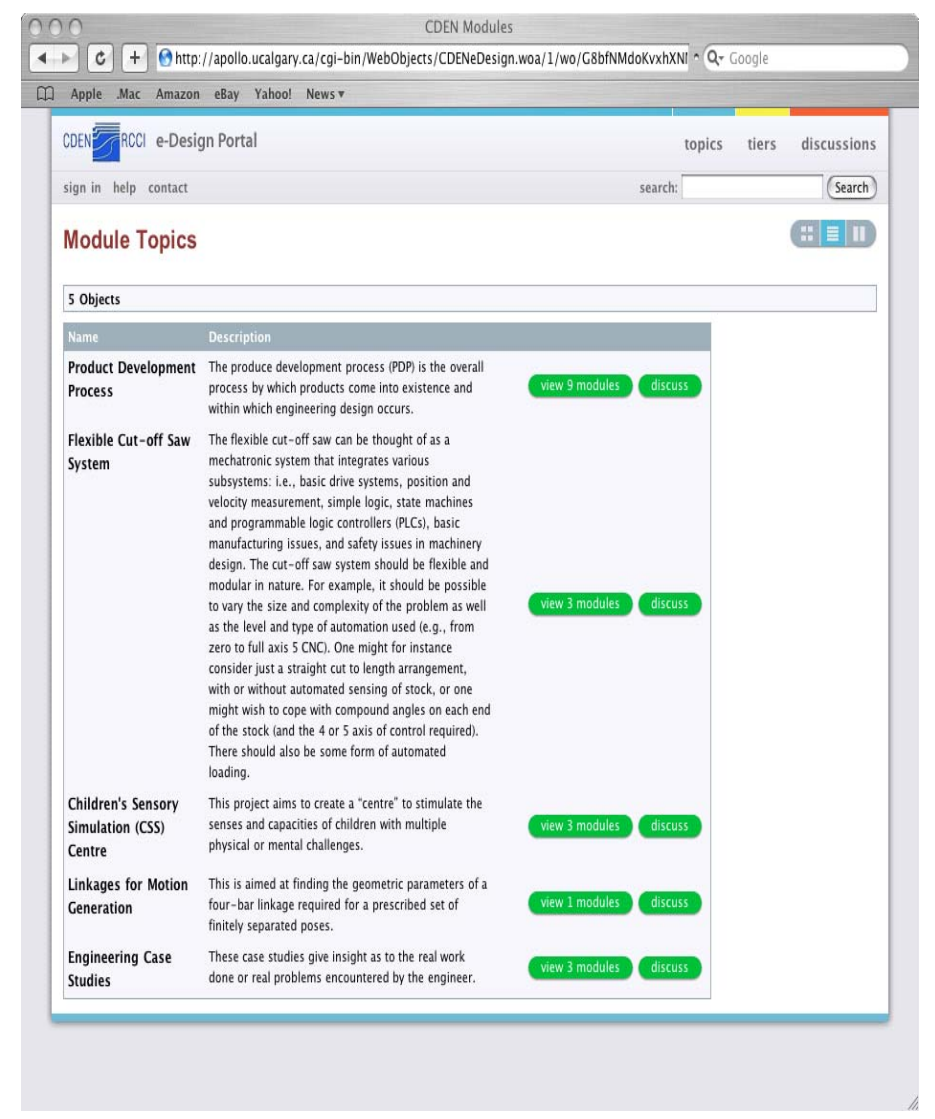

Figure 3. An e-Design Portal topic page

engineering design courseware. As noted previously, considerable design courseware has already been developed by members of CDEN, however to this date there has not been a single online repository for this material. The three-tiered module classification system and the searchable database described in this paper show promise as a solution to this problem.

The first draft of the CDEN e-Design Portal is scheduled to go online in early July 2005 . At that time, existing courseware (i.e., CDEN Design Modules) from CDEN members will be added to the database and made available for review. Based on the review of this material, the module guidelines will be refined in order to provide a relatively consistent interface to users. As well, the impact of the e-Design Portal on engineering design education in Canada will then be investigated through CDEN partner universities across Canada.

\section{Acknowledgements}

The programming of the e-Design Portal was performed by Julian Wood and King Huang of the
University of Calgary, Learning Commons. CDEN modules included in the e-Design Portal at the time of writing were contributed by members of the Canadian Design Engineering Network.

The author wishes to thank the attendees of the CDEN Workshop on Developing Web-based Design Modules (16 October, 2004, University of Toronto) and especially Dr. Paul Gauvreau, University of Toronto, and Dr. Paul Stuart, École Polytechnique, for their help with the workshop organization.

The author also wishes to thank the Natural Sciences and Engineering Research Council of Canada and the University of Calgary, Faculty of Engineering for their generous support of this research.

\section{References}

[1] Yellowley, I. and P. Gu, "Design directed engineering education", Proceedings of the Inaugural CDEN Design Conference, Montreal, July 29-30, 2004.

[2] Angeles, J., R. Britton, L. Chang, F. Charron, P. Gregson, P. Gu, P. Lawrence, W. Stiver, D. Strong, P. Stuart, B. Thompson, "The engineering design 


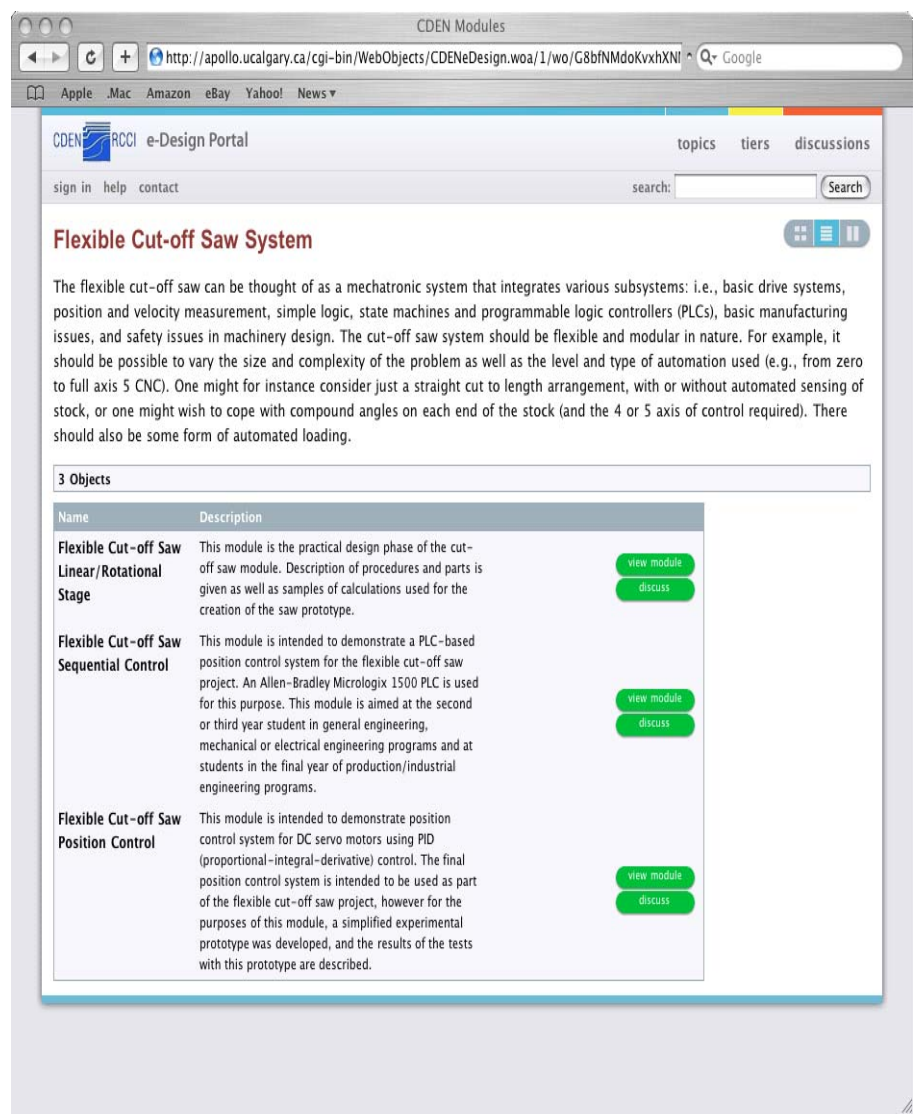

Figure 4. e-Design Portal inspect modules page

competency", Proceedings of the Inaugural CDEN Design Conference, Montreal, July 29-30, 2004.

[3] Canadian Design Engineering Network, Web Site, http://www.cden.ca/, 2005.

[4] Clark, R.C. and R.E. Mayer, e-Learning and the Science of Instruction, Pfeiffer, 2003.

[5] Yellowley, I., R.D. Venter, F.A. Salustri, "The Canadian design engineering network (CDEN/RCCI): sharing engineering design educational tools within thirty three schools of engineering in Canada", Proceedings of the 8th World Conference on Continuing Engineering Education, 2001.
[6] Hammersley, B., Content Syndication with RSS, O'Reilly \& Associates, 2003.

[7] Blackboard, Web Site, http://www.blackboard.com/, 2005.

[8] Brennan, R.W, M. Eggermont and M. Shams, "Towards engineering design courseware", Proceedings of the Second CDEN International Conference on Design Education, Innovation, and Practice, Kananaskis, Canada, July 18-20, 2005.

[9] CDEN e-Design Portal, Web Site, http://apollo.ucalgary.ca/cden, 2005.

[10] Learning Commons, Web Site, http://commons.ucalgary.ca/, 2005. 


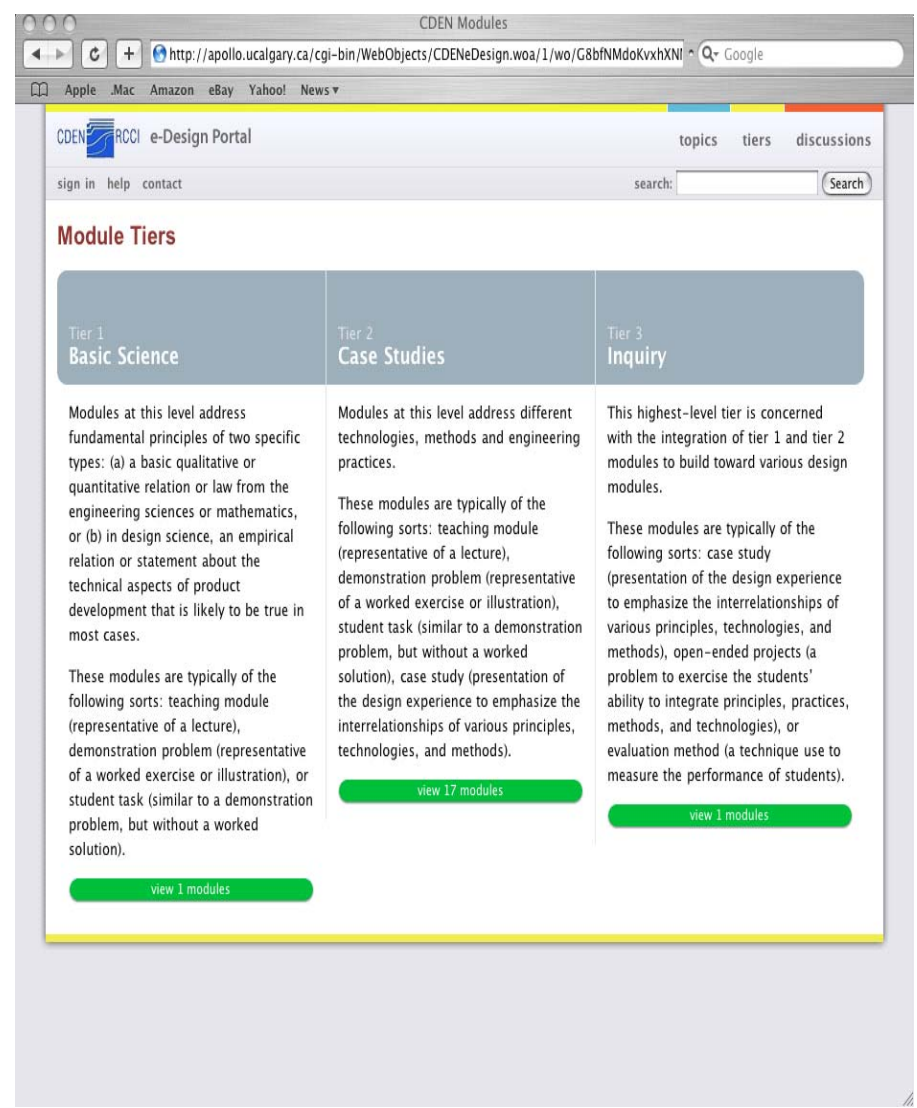

Figure 5. e-Design Portal inspect tiers page 


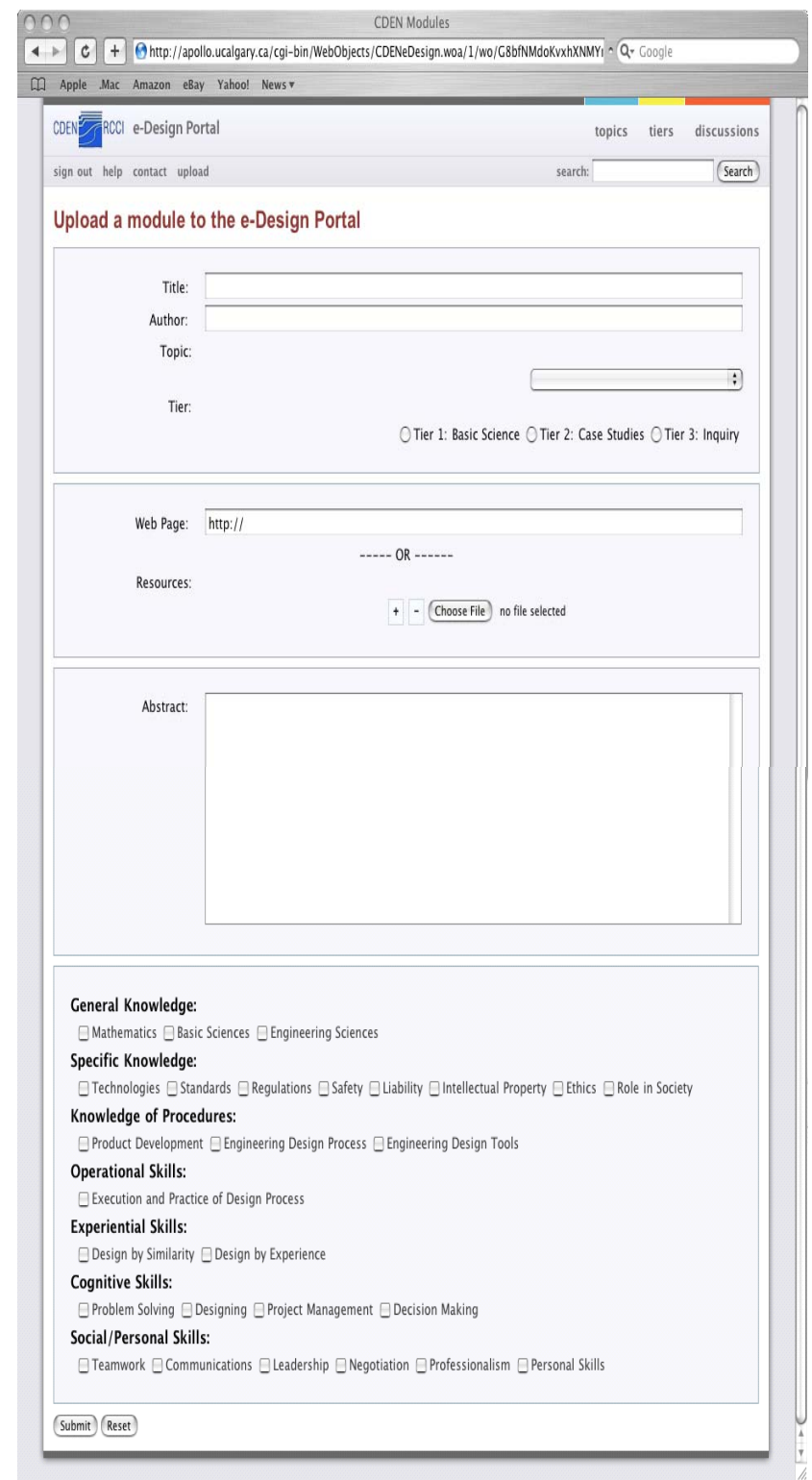

Figure 6. Uploading modules to the e-Design Portal 